\title{
Spartan annual meeting: Denver, Colorado
}

\author{
Luis R. Espinoza ${ }^{1}$
}

Published online: 13 April 2016

(C) International League of Associations for Rheumatology (ILAR) 2016

The annual meeting of SPARTAN (Spondyloarthritis Research and Treatment Network) was held in Denver, Colorado on July 25 and 26, 2015, and it was preceded by a combined SPARTAN-SAA (Spondylitis Association of American) MRI workshop held on July 24th. SPARTAN is a growing network of health care professionals in North America, Mexico, and other Latin American countries dedicated to foster research, education, awareness, and therapy of spondyloarthritis. This group of arthritides characteristically involves the axial skeleton, although peripheral joints and extra-articular manifestations may also be exhibited; it is highly prevalent among younger individuals in whom it constitutes a frequent cause of long-standing lower back pain leading to a significant impact in quality of life, functional impairment, and disability if left untreated $[1,2]$. The pathogenesis of spondyloarthritis remains to be elucidated, although considerable progress has been made in recent years in terms of early diagnosis and newer and highly effective therapeutic modalities.

This issue of Clinical Rheumatology highlights 6 papers presented at the annual meeting. It was an intense 3-day affair devoted to basic, clinical, diagnostic, and therapeutic discussion concerning spondyloarthritis by a group of experts.

Drs. Khan and Akkoc in the opening article present a provocative proposal to modify existent ASAS classification criteria for axial spondyloarthritis (AS). They provide an overview of the classification criteria of AS beginning with the first criteria set developed at the rheumatic disease conference

Luis R. Espinoza

luisrolan@msn.com

1 Section of Rheumatology, LSU Health Sciences Center at New Orleans, 1542 Tulane Avenue, New Orleans, LA 70112, USA in Rome in 1961 and thoroughly describe the newer classification criteria introduced by the Assessment of Spondyloarthritis International Society (ASAS) that have had a significant impact on the terminology of this group of arthritides, with the terms axSpA that includes ankylosing spondylitis (AS) and non-radiographic axSpA (nr-axSpA). They suggest that the multi-arm construct of the ASAS criteria is a potential source of heterogeneity that thus reduces their utility. They provide evidence suggesting that it is time to modify the ASAS criteria for axSpA and also propose steps that may improve the validity of these criteria.

The next 3 topics present and discuss important basic mechanistic aspects in the development of spondyloarthritis that provide newer insights into their pathogenesis. First, Smith discusses the potential role of the unfolded protein response in axial spondyloarthritis. HLA-B27 MHC class I allele remains the strongest genetic risk factor identified in the great majority of AS patients. In addition to its role in antigen presentation, HLA-B27 possesses unique biochemical characteristics that may contribute to disease pathogenesis. HLAB27 folds slowly and misfolds during its biosynthesis in the endoplasmic reticulum (ER), and this unfolded protein response (UPR) might lead to ER stress and may also increase inflammatory cytokines and affect autophagy. Evidence for the relevance of an HLA-B27-related UPR to the development of spondyloarthritis in animal models and humans is discussed.

Next, Ciccia and Haroon introduced us to a newer concept, autophagy, and how it might relate to the pathogenesis of $\mathrm{SpA}$, and as such it closely interacts with the previous discussion of the role on unfolded protein response. First, they introduce the concept, discussed the types and its mechanism of action. Then, they briefly present an overview of the role of autophagy in health and disease and conclude by discussing autophagy in rheumatic diseases and particularly AS. Findings discussed appeared to suggest that autophagy could 
be a critical link in the pathogenesis of AS and that further investigation is needed to fully elucidate its role in the pathogenesis of AS and related disorders.

Cytokines play a critical role in the pathogenesis of spondyloarthritis, and this topic is discussed by Siba and Smriti Raychaudhuri with emphasis on the IL-23-IL-17 axis, especially in the inflammatory and proliferative cascades of SpA. They describe a series of experiments using animal models and human tissues in which it was demonstrated that IL-23-induced Th17 cytokines (IL-17 and IL-22) can contribute to the development of psoriasis plaques, pannus formation in the joint, joint erosion, and new bone formation. These findings are of utmost relevance in view of the newer IL-23/ IL-17-targeted biological therapies that have shown great promise in the management of SpA.

Gensler discusses the role of physical activity in axial spondyloarthritis, which together with recreational activity are the cornerstone of non-pharmacologic therapy in the management of these disorders. The review provides a comprehensive overview of beneficial and non-beneficial physical activities including occupational therapy and also includes an examination of animal model-based evidence and epidemiologic studies on physical activity in axSpA. Evidence presented supports the notion that in general physical activity is associated with better outcomes in patients with AS. However, it is not clear or well-defined what component of this type of physical activity is directly impacting clinical manifestations and potentially inflammation.

Lastly, Maksymowych provides a comprehensive overview of the role of MRI in the evaluation of spondyloarthritis.
Clinicians, including rheumatologists, are not completely familiarized with the technology and interpretation of this important tool, which can provide important diagnostic information, monitoring of disease activity, and response to therapy. This review addresses five key points: when should MRI be indicated during the diagnostic investigation, type of MRI ordered, types of lesions seen in early disease and their diagnostic utility, how can MRI be used to select patients for appropriate therapeutic intervention, and lastly, define its role in the assessment of patients with established SpA. Current evidence suggest that MRI is a valuable tool in the assessment of patients with $\mathrm{SpA}$ and that clinicians including rheumatologists taking care of SpA patients should become familiar with this technique and learned its basic uses, limitations, and developments. MRI findings form part of the ASAS classification criteria and are also taken into consideration by regulatory agencies in the final approval for the use of biologic agents in the early stages of axSpA.

\section{References}

1. Bautista-Molano W, Landewe RB, Londono J, Romero- Sanchez C, Valle-Onate R, van der Heijde D (2016) Analysis and performance of various classification criteria sets in a Colombian cohort of patients with spondyloarthritis. Clin Rheumatol. doi:10.1007/s10067-0163184-2

2. Erbil J, Espinoza LR (2015) Nonradiographic axial spondyloarthritis background and confounding factors of this new terminology: an appraisal. Clin Rheumatol 34:407-11 